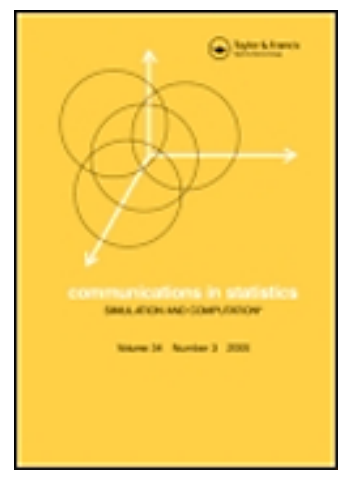

\title{
An adaptive test for the two-sample scale problem based on U-statistics
}

\begin{tabular}{|c|c|}
\hline Journal: & Communications in Statistics - Simulation and Computation \\
\hline Manuscript ID: & LSSP-2010-0136 \\
\hline Manuscript Type: & Original Paper \\
\hline $\begin{array}{r}\text { Date Submitted by the } \\
\text { Author: }\end{array}$ & 14-May-2010 \\
\hline Complete List of Authors: & $\begin{array}{l}\text { Koessler, Wolfgang; Humboldt-Universitaet zu Berlin, Informatik } \\
\text { Kumar, Narinder; Panjab University Chandigarh, Statistics }\end{array}$ \\
\hline Keywords: & $\begin{array}{l}\text { Mood-test, Klotz-test, asymptotic efficacy, asymptotic power, } \\
\text { simulation study, tailweight, skewness }\end{array}$ \\
\hline Abstract: & $\begin{array}{l}\text { For the two-sample scale problem with continuous data we } \\
\text { consider a } \\
\text { general class of tests, all members of it are based on U-statistics. } \\
\text { The asymptotic efficacies are investigated in detail. } \\
\text { We construct an adaptive test where all statistics involved are } \\
\text { suitably chosen U-statistics. } \\
\text { It is shown that the proposed adaptive test } \\
\text { has good asymptotic and finite sample power properties. }\end{array}$ \\
\hline \multicolumn{2}{|c|}{$\begin{array}{l}\text { Note: The following files were submitted by the author for peer review, but cannot be converted } \\
\text { to PDF. You must view these files (e.g. movies) online. }\end{array}$} \\
\hline KoesslerKumar.zip & \\
\hline
\end{tabular}

\section{SCHOLARONE ${ }^{\text {M }}$ Manuscripts}




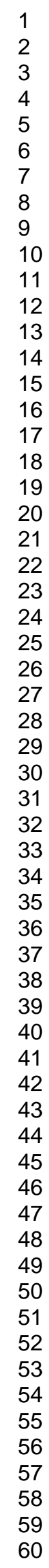

14

15

7

18

0

21

3 6

60 


\title{
An adaptive test for the two-sample scale problem based on U-statistics
}

\author{
W. Kössler, Humboldt-Universität zu Berlin, \\ koessler@informatik.hu-berlin.de \\ N. Kumar, Panjab University Chandigarh, \\ nkumar67@yahoo.com
}

\begin{abstract}
For the two-sample scale problem with continuous data we consider a general class of tests, all members of it are based on U-statistics. The asymptotic efficacies are investigated in detail. We construct an adaptive test where all statistics involved are suitably chosen U-statistics. It is shown that the proposed adaptive test has good asymptotic and finite sample power properties.
\end{abstract}

Keywords: Mood-test, Klotz-test, asymptotic efficacy, asymptotic power, simulation study, tailweight, skewness.

Subject classification: $62 \mathrm{G} 10,62 \mathrm{G} 20$

\section{Corresponding author:}

Wolfgang Kössler, Humboldt-Universität zu Berlin, Institut für Informatik Rudower Chaussee 25, D-12489 Berlin

Running title: Adaptive scale test based on U-statistics 


\section{Introduction}

Let $X_{1}, \ldots, X_{n_{1}}$ and $Y_{1}, \ldots, Y_{n_{2}}$ be independent random samples from populations with absolutely continuous distribution functions $F(x)$ and $F\left(\frac{x}{e^{\vartheta}}\right)$, $\vartheta \in \mathbf{R}$, respectively. We wish to test

$$
H_{0}: \quad \vartheta=0
$$

against

$$
H_{1}: \quad \vartheta \neq 0 .
$$

The Klotz test and the Mood test are the most familiar nonparametric tests for this problem. In the present paper we will consider a class of tests based on U-statistics. This interesting class of tests has drawn considerable attraction in the literature, cf., e.g. Deshpande and Kochar (1982), Shetty and Govindarajulu (1988), Kumar (1997), Xie and Priebe (2000, 2002), John and Priebe (2007) for the location problem.

For the scale problem there are also some papers, cf. e.g. Sukhatme (1957, 1958), Tamura (1960, 1962, 1966), Deshpande and Kusum (1985), Kusum (1985), Kochar and Gupta (1986).

The statistics of Kochar and Gupta are special cases of our class, and they again are generalizations of that used by Tamura $(1960,1962,1966)$. From the ARE considerations we will see that our statistics $U_{5: 1}$ and $U_{8: 1}$ (and $U_{5: 2}$ for densities with very long tails) outperform the other statistics (see section 3). A somewhat different class of scale tests based on U-statistics was proposed by Joshi and Shetty (2008). However, as we will see, some of their statistics are asymptotically equivalent to some of ours.

In section 2 a general class $U_{k: i}$ of U-statistics is defined. Scale alternatives of the form $\vartheta=\theta_{N}=\theta / \sqrt{N}, N=n_{1}+n_{2}$, are considered and the asymptotic efficacies of the tests based on $U_{k: i}$ are compared in Section 3. It is shown that there are different tests of this type which are efficient for densities with short, medium or long tails, respectively. For example, the test based on $U_{8: 1}$ is efficient for densities with short tails, and that based on $U_{6: 3}$ is efficient for densities with very long tails. However, the practising statistician has generally no clear idea on the underlying density, thus he/she should apply an adaptive test which takes into account the given data set. In Section 4 two versions of such an adaptive test are proposed, one of them is distributionfree. The adaptive tests first classify the underlying distribution with respect to some measures like that of tailweight and then select an appropriate test 
based on U-statistics. Our adaptive test is compared briefly with adaptive tests based on linear rank tests in Section 5. In Section 6 a simulation study is performed and the finite sample power is compared with the asymptotic power. A data example is provided in Section 7. We give some conclusions in Section 8.

\section{Test statistics}

We consider the class of U-statistics, which was proposed by Kumar (2008). Let $k, 1 \leq k \leq \min \left(n_{1}, n_{2}\right)$, and $i, i \leq \frac{k+1}{2}$, be fixed integers. Define

$\Phi_{i}\left(x_{1}, \ldots, x_{k}, y_{1}, \ldots, y_{k}\right)= \begin{cases}1 & \text { if } x_{(i) k}<y_{(i) k} \text { and } x_{(k-i+1) k}<y_{(k-i+1) k} \\ -1 & \text { if } x_{(i) k}>y_{(i) k} \text { and } x_{(k-i+1) k}>y_{(k-i+1) k} \\ 0 & \text { otherwise, }\end{cases}$ where $x_{(i) k}$ is the $i$ th order statistic in a subsample of size $k$ from the $X$ sample (and likewise for $y^{\prime}$ 's). Let $U_{k: i}$ be the U-statistic associated with kernel $\Phi_{i}$, i.e.

$$
U_{k: i}=\frac{n_{1} n_{2}}{\left(\begin{array}{c}
n_{1} \\
k
\end{array}\right) \cdot\left(\begin{array}{c}
n_{2} \\
k
\end{array}\right)} \sum \Phi_{i}\left(X_{r_{1}}, \ldots, X_{r_{k}}, Y_{s_{1}}, \ldots, Y_{s_{k}}\right)
$$

where the summation extends over all possible combinations $\left(r_{1}, \ldots, r_{k}\right)$ of $k$ integers from $\left\{1, \ldots, n_{1}\right\}$ and all possible combinations $\left(s_{1}, \ldots, s_{k}\right)$ of $k$ integers from $\left\{1, \ldots, n_{2}\right\}$. The null hypothesis $H_{0}$ is rejected in favour of $H_{1}$ for large values of $U_{k: i}$.

Remark: For $i=1$ or $i=k$ we have the Kochar-Gupta test (cf. Kochar and Gupta, 1986).

There is also a rank representation of the $U_{k: i}$. Let $R_{(s)}$ be the rank of $Y_{(s)}$ in the joint ranking of the $X$-sample and $Y$-sample, where $Y_{(s)}$ is the $s$ th order statistics of the $Y$-sample. Then

$$
\begin{array}{r}
\frac{\left(\begin{array}{c}
n_{1} \\
k
\end{array}\right)\left(\begin{array}{c}
n_{2} \\
k
\end{array}\right)}{n_{1} n_{2}} U_{k: i}=\sum_{s=i}^{n_{2}} \sum_{j=i}^{k}\left(\begin{array}{c}
s-1 \\
i-1
\end{array}\right)\left(\begin{array}{c}
n_{2}-s \\
k-i
\end{array}\right)\left(\begin{array}{c}
R_{(s)}-s \\
j
\end{array}\right)\left(\begin{array}{c}
n_{1}+s-R_{(s)} \\
k-j
\end{array}\right) \\
-\sum_{s=k-i+1}^{n_{2}} \sum_{j=k-i+1}^{k}\left(\begin{array}{c}
s-1 \\
k-i
\end{array}\right)\left(\begin{array}{c}
n_{2}-s \\
i-1
\end{array}\right)\left(\begin{array}{c}
R_{(s)}-s \\
j
\end{array}\right)\left(\begin{array}{c}
n_{1}+s-R_{(s)} \\
k-j
\end{array}\right) .
\end{array}
$$


Let

$$
\begin{aligned}
\varphi_{1,0}^{(i)}(x) & =\mathbf{E} \Phi_{i}\left(x, X_{2}, \ldots, X_{k}, Y_{1}, \ldots, Y_{k}\right) \\
\varphi_{0,1}^{(i)}(y) & =\mathbf{E} \Phi_{i}\left(X_{1}, \ldots, X_{k}, y, Y_{2}, \ldots, Y_{k}\right) \\
\zeta_{1,0}^{(i)} & =\operatorname{Var}\left(\varphi_{1,0}^{(i)}(X)\right) \\
\zeta_{0,1}^{(i)} & =\operatorname{Var}\left(\varphi_{0,1}^{(i)}(Y)\right),
\end{aligned}
$$

where $\mathbf{E}$ and Var denote the expectation and variance respectively. Moreover, let $F_{(i) k}($.$) be the cumulative distribution function of the i$ th order statistics of a sample of size $k$.

Proposition 2.1 (cf. Kumar, 2008) Under assumptions $N \rightarrow \infty, n_{1} / N \rightarrow$ $\lambda, 0<\lambda<1$ the limiting distribution of $N^{1 / 2}\left(U_{k: i}-\eta_{k, i}\right) / \sigma_{k, i}$ is standard normal, where expectation $\eta_{k, i}=\mathbf{E} U_{k: i}$ and variance $\sigma_{k, i}^{2}=\operatorname{Var}\left(U_{k: i}\right)$ have the forms

$$
\begin{aligned}
\eta_{k, i}= & n_{1} n_{2}\left(i\left(\begin{array}{c}
k \\
i
\end{array}\right)\right)^{2}\left(\int_{-\infty}^{\infty} y(F(y))^{2 i-2}(1-F(y))^{2 k-2 i} f^{2}(y) d y-\right. \\
& \int_{-\infty}^{\infty} y(F(y))^{2 k-2 i}\left(1-F(y)^{2 i-2} f^{2}(y) d y\right) \\
\sigma_{k, i}^{2}= & n_{1}^{2} n_{2}^{2}\left(\frac{k^{2} \zeta_{10}^{(i)}}{\lambda}+\frac{k^{2} \zeta_{01}^{(i)}}{1-\lambda}\right) .
\end{aligned}
$$

Remark: Under $H_{0}$ we have $\eta_{k, i}=0$ and

$$
\sigma_{k, i}^{2}=n_{1}^{2} n_{2}^{2} k^{2} \frac{\rho_{k, i}}{\lambda(1-\lambda)}
$$

where $\rho_{k, i}$ depends on $k$ and $i$ only. The expression for $\rho_{k, i}$ is rather long, that is why we do not write it out. It can be found in Kumar (2008).

\section{The asymptotic efficacies}

The asymptotic (Pitman-) efficacies AE of the statistics $U_{k: i}$ under the alternative $\theta_{N}=N^{-1 / 2} \cdot \theta$ are given by

$$
A E\left(U_{k: i} \mid f\right)=\lambda(1-\lambda) \cdot C_{k, i}^{2}(f),
$$


where $f(\cdot)$ denotes the probability density function belonging to the c.d.f. $F(\cdot)$ and

$$
\begin{aligned}
C_{k, i}(f)=\frac{\left(\left(\begin{array}{c}
k \\
i
\end{array}\right) i\right)^{2}}{\left(k^{2} \rho_{k, i}\right)^{1 / 2}} \cdot\left(\int_{-\infty}^{\infty} x(F(x))^{2 i-2}(1-F(x))^{2 k-2 i} f^{2}(x) d x-\right. \\
\left.\int_{-\infty}^{\infty} x(F(x))^{2 k-2 i}(1-F(x))^{2 i-2} f^{2}(x) d x\right) .
\end{aligned}
$$

Note that the asymptotic efficacy is defined by the limit of $\eta_{k, i}^{2} / \sigma_{k, i}^{2}$, cf. Noether (1955). Since only the factor $C_{k, i}(f)$ is of interest here we refer to this factor if we say AE.

Remark: It may be shown that the Mood test is asymptotically equivalent to the test $U_{2: 1}$.

Before we continue with the investigation of our class of U-statistics, we will compare the asymptotic efficacies of our tests with that of other tests based on U-statistics. First, let us note that the statistics $S J(2 k-1, r, 1)$ with $r=1$ of Joshi and Shetty are asymptotically equivalent to our statistics $U_{k: 1}$. (Joshi and Shetty consider only the case $r=1$.) In Table 1 we find the AE of the tests of Sukhatme (1958), Kusum (1985), three of the best variants of Joshi and Shetty (2008) (abbreviated by $S J(5,1), S J(9,1), S J(5,2)$ ) as well as of the tests $U_{8: 1}, U_{2: 1}$ and $U_{5: 2}$ for five densities, the uniform, logistic, Cauchy, DE, and normal. The AE of the best of these eight tests is written in bold style, respectively. Missing entries are not relevant or not computed. Most computations are done with the Mathematica package, few could be done analytically. From Table 1 we see that Sukhatme, Kusum, SJ $(5,1)$ and $\mathrm{SJ}(5,2)$ are in no cases the best tests. Recall that $S J(9,1)$ is asymptotically equivalent to $U_{5: 1}$.

Table 1 about here

For further investigations we have to make some restrictions. We restricted the choice of $k$ at first to $k \leq 6$ ourselves just for illustrative purpose. Of course, computations can be made for values of $k$ beyond that.

We compute the asymptotic Pitman efficacies for all tests $U_{k: i}$ with $1 \leq$ $i \leq \frac{k}{2}, k \leq 6$. Obviously, for $i=\frac{k+1}{2}$ we have $C_{k, i}=0$. Values of the factors $C_{k, i}^{2}(f)$ for various densities are, together with that of the well established Klotz test presented in Table 2.

The L-DE density was proposed by Policello and Hettmansperger (1976), 
the U-L by Gastwirth (1965), the RST is named after Ramberg, Schmeiser and Tukey, cf. Ramberg and Schmeiser $(1972,1974), \mathrm{CN}(\epsilon, \sigma)$ is the scale contaminated normal with contaminating proportion $\epsilon$, and the skew-logistic is proposed by Nadarajah (2009).

The bold entries denote, for the given density, the asymptotically best test among the considered tests. (If the Klotz-test is the asymptotically best, it is denoted in italics.) If no test is bold, then one of the tests $U_{7: 1}, U_{8: 1}$, or $U_{9: 1}$ has larger AE-values (see below), and the corresponding entry is bold in Table 3 .

On the first view we see that the columns for $U_{8: 1}, U_{5: 1}$ and $U_{2: 1}$ have the most bold entries. This observation gives rise to the idea to use these few statistics in our adaptive test.

To get a closer idea how to classify symmetric densities we apply the method of Hall and Joiner (1982). The content of information in the asymptotic efficacy matrix is analysed by a principal component analysis where the densities are the observations and the efficacies of the $U_{k: i}$ are the variables. The first principal component explains already $98 \%$ of the variability. For better visibility we display in Figure 1 the values of the first two principal components (Factors one and two). Nearly symmetric densities with short tails are denoted by a green plus, those with short-medium and long-medium tails by a cyan $\mathrm{X}$ and a blue star, respectively, and that with very long tails with a red dot.

On the left side we have densities with long tails, in the centre that with medium tails, and on the right that with short tails. For an exact definition what we understand by very long, (long- and short-) medium and short tails see below. On the first view we see that the $A E\left(U_{k: i}\right)$ classify the densities according to their tailweight.

Since very often the tests $U_{6: 1}$ and $U_{5: 1}$ are asymptotically the best it might be desirable to investigate also the tests $U_{7: 1}, U_{8: 1}$, and $U_{9: 1}$. From this investigation we found that it might be a good idea to choose the test $U_{8: 1}$ for short tail densities. AE values for the test $U_{8: 1}$ are, togehter with that of $U_{5: 1}$ given in Table 3 .

When we consider skew densities we have to differ between densities defined on the whole real line (such as the Gumbel or the skew-logistic) and that starting at some point, e.g. at zero (as it is, e.g. for time data). Skew densities of the former group may be included in the scheme designed for symmetric densities. For the latter the considered U-tests are bad (cf. Table 4). Much better in this case are tests that are designed for testing locations, 
e.g. the Mann-Whitney test (or another U-test, cf. Kössler, Kumar, 2008). In Table 4 we included the Mann-Whitney test, denoted by $U_{1,1}$. The location U-test $U_{2,1}$ of Kössler and Kumar (2008) seems to be asymptotically slightly better than the Mann-Whitney test, but for convenience of the reader we preferred the latter test here.

Insert Figure 1 about here

\section{Adaptive test}

At first we have to decide whether we have a density with some starting point (which is generally zero) or not. In the first case we apply a location test on the data, such as the Mann-Whitney-Wilcoxon test. Alternatively we may perform a logarithmic transformation and then apply an adaptive location test, e.g. that of Kössler and Kumar (2008) which is also based on U-statistics.

Assume now that we have a symmetric density or a skew density that is defined on the whole real line. We apply the concept of Hogg (1974), that is, to classify at first the type of the underlying density with respect to one measure of tailweight $\hat{t}$, which is defined by

$$
\hat{t}=\frac{\hat{Q}(0.95)-\hat{Q}(0.05)}{\hat{Q}(0.85)-\hat{Q}(0.15)}
$$

where $\hat{Q}(u)$ is the so-called classical quantile estimate of $F^{-1}(u)$,

$$
\hat{Q}(u)=\left\{\begin{array}{lll}
X_{(1)}-(1-\delta) \cdot\left(X_{(2)}-X_{(1)}\right) & \text { if } \quad u<1 /(2 \cdot N) \\
(1-\delta) \cdot X_{(j)}+\delta \cdot X_{(j+1)} & \text { if } \quad \frac{1}{2 \cdot N} \leq u \leq \frac{2 \cdot N-1}{2 \cdot N} \\
X_{(N)}+\delta\left(X_{(N)}-X_{(N-1)}\right) & \text { if } & u>(2 \cdot N-1) /(2 \cdot N)
\end{array}\right.
$$

where $\delta=N \cdot u+1 / 2-j$ and $j=\lfloor N \cdot u+1 / 2\rfloor$.

In Table 5, together with the $\mathrm{AE}$ of the adaptive tests, the values of the corresponding theoretical measures $t$ for various selected densities are presented.

Comparing Tables 2 and 3 with the tailweight measures roughly we see that the tests $U_{4: 1}, U_{5: 1}$ and $U_{6: 1}$ are asymptotically good tests for symmetric 
densities with small to medium tailweight, $U_{3: 1}$ and $U_{2: 1}$ for symmetric densities with longer tailweight, $U_{4: 2}, U_{5: 2}, U_{6: 2}$ and $U_{6: 3}$ for symmetric densities with very large tailweight.

From Table 3 we see that for medium tails the tests $U_{5: 1}$ or $U_{6: 1}$ are the best choices whereas for short tails the tests $U_{8: 1}$ and $U_{9: 1}$ are the best.

The reasoning of the last two sections gives rise to the following adaptive test.

Define regions $E_{1}, \ldots, E_{4}$ which are based on the selector statistic $\hat{t}$ which is given by (1),

$$
\begin{aligned}
& E_{1}=\{\hat{t}<1.61\} \quad \text { "short tails" } \\
& E_{2}=\{1.61 \leq \hat{t}<1.93\} \quad \text { "light medium tails" } \\
& E_{3}=\{1.93 \leq \hat{t} \leq 2.5\} \quad \text { "long tails" } \\
& E_{4}=\{\hat{t}>2.5\} \quad \text { "very long tails" }
\end{aligned}
$$

The cutoff values of the regions are determined in such a way that the vast majority of densities is classified correctly, i.e. they fall in the class that has the highest asymptotic power (cf. Tables 2 and 3 with Table 5). For example, the normal (tailweight $t=1.59$, cf. Table 5 ) is classified to $E_{1}$, and the test $U_{8: 1}$, which is the best among the considered tests (cf. Tables 2 and 3 ), is performed. The logistic is mapped to region $E_{2}$ and the test $U_{5: 1}$ is performed. Similar observations for the other densities lead to the given cutoff values. In few cases, if the classification doesn't be correct, then the efficacy loss is very small in almost all cases. In Tables 2 and 3 the chosen test is underlined if it is not already the (bold) best.

Now, we propose the Adaptive test $A$ which is based on the four Ustatistics $U_{8: 1}, U_{5: 1}, U_{2: 1}$, and $U_{5,2}$. We denote the tests by (8:1), (5:1), (2:1) and $(5: 2)$, respectively.

$$
A=A(\hat{t})=\left\{\begin{array}{lll}
(8: 1) & \text { if } \quad \hat{S} \in E_{1} \\
(5: 1) & \text { if } \quad \hat{S} \in E_{2} \\
(2: 1) & \text { if } \quad \hat{S} \in E_{3} \\
(5: 2) & \text { if } \quad \hat{S} \in E_{4}
\end{array}\right.
$$

In Figure 2 the corresponding adaptive scheme is given.

Insert Figure 2 about here 
The two-stage procedure defined above is asymptotically distribution-free since the selector statistic $\hat{t}$ is based on the order statistic only and the Ustatistics are based on the ranks only.

The Adaptive test $A$ is only asymptotically distribution-free because asymptotic critical values are used in the adaptive scheme.

Proposition 4.1 Let $\left\{\theta_{N}\right\}$ be a sequence of 'near' alternatives with $\sqrt{N} \theta_{N} \rightarrow$ $\theta$. The asymptotic power function of the Adaptive test $A$ equals

$$
\beta(\theta)= \begin{cases}1-\Phi\left(z_{1-\alpha}-\sqrt{A E\left(U_{8: 1} \mid f\right)} \cdot \theta\right) & \text { if } f \in E_{1} \\ 1-\Phi\left(z_{1-\alpha}-\sqrt{A E\left(U_{5: 1} \mid f\right)} \cdot \theta\right) & \text { if } f \in E_{2} \\ 1-\Phi\left(z_{1-\alpha}-\sqrt{A E\left(U_{2: 1} \mid f\right)} \cdot \theta\right) & \text { if } f \in E_{3} \\ 1-\Phi\left(z_{1-\alpha}-\sqrt{A E\left(U_{5: 2} \mid f\right)} \cdot \theta\right) & \text { if } f \in E_{4}\end{cases}
$$

Proof. Let be $h=1$ if $(k: i)=(8: 1), h=2$ if $(k, i)=(5: 1), h=3$ if $(k, i)=(2: 1), h=4$ if $(k, i)=(5: 2)$ Let be $T_{1}=U_{8: 1}, T_{2}=U_{5: 1}, T_{3}=U_{2: 1}$, $T_{4}=U_{5: 2}$. The proposition follows from the total probability theorem and from the consistency of the selector statistics, i.e.

$$
\begin{aligned}
\beta(\theta) & =\sum_{h=1}^{4} P_{\theta}\left(T_{h}>c_{\alpha h} \mid T_{h} \text { chosen }\right) \cdot P_{\theta}\left(T_{h} \text { chosen }\right) \\
& =\sum_{h=1}^{4}\left(1-\Phi\left(z_{1-\alpha}-\sqrt{A E\left(T_{h} \mid f\right)} \cdot \theta\right)+o(1)\right) \cdot\left\{\begin{array}{l}
1+o(1) \quad \text { if } f \in E_{h} \\
o(1) \quad \text { else }
\end{array}\right. \\
& \sim 1-\Phi\left(z_{1-\alpha}-\sqrt{A E\left(T_{h} \mid f\right)} \cdot \theta\right) \quad \text { if } \quad f \in E_{h},
\end{aligned}
$$

where $c_{\alpha h}$ is the $(1-\alpha)$-quantile of the asymptotic null distribution of $T_{h}$.

The Adaptive test $A(\hat{t})$ is based on selector statistics computed from the pooled sample. However, location differences may effect the estimates of tailweight and skewness. That is why we also consider a modification $A\left(\hat{t}^{*}\right)$ of the adaptive test, where tailweights are estimated from the single samples. Let $\hat{t}_{i}, i=1,2$ be statistics of the form (1) for tailweight. Applying the $A\left(\hat{t}^{*}\right)$-test the selector statistic $\left.\hat{t}^{*}\right)$ with

$$
\hat{t}^{*}=\frac{n_{1}}{N} \hat{t}_{1}+\frac{n_{2}}{N} \hat{t}_{2}
$$

is used instead of $\hat{t}$. This procedure is also asymptotically distribution-free. However, it is not distribution-free also if the exact critical values are used. 
This property is since the selector statistic is no longer based on the pure order statistic.

For various densities asymptotic power functions (together with finite power functions) are given in Figures 3 and 4.

The blue dotted line is for $U_{8: 1}$, the violet short-dashed line for $U_{5: 1}$, the green long-dashed line for $U_{2: 1}$, the red dashed-dotted line for $U_{5: 2}$ (and the black continuous line for the adaptive test).

\section{Comparison to adaptive tests based on lin- ear rank statistics}

Restrictive adaptive tests for the two-sample scale problem based on linear rank tests are proposed by Rünstler (1987) and Kössler (1994), as well as by Hall and Padmanabhan (1997), cf. also Büning (1991). All of them are based on the concept of Hogg (1974), and they use few linear rank statistics, with some scores. Rünstler (1987) considered only symmetric densities, and he used the Gastwirth scores (for short tails), Mood scores (for medium tails), and Cauchy scores (for long tails). Kössler (1994) used the Klotz scores (for symmetric densities with short tails), logistic scores (for symmetric densities with medium tails), Ansari-Bradley scores (for long-tail densities), the Savage and Wilcoxon scores for skew densities with short or medium tails, respectively. Hall and Padmanabhan (1997) performed a transformation to handle unequal locations and applied the Klotz-test and the Wilcoxon test to the transformed data (their Adaptive procedure II). For the estimation of tailweight Rünstler (1987) and Hall and Padmanabhan (1997) used integral measures whereas Kössler (1994) used quantile measures for his tailweight and skewness estimates.

Let us denote the adaptive test of Kössler (1994) by $B(\hat{S})$, where $\hat{S}=$ $(\hat{t}, \hat{s})$ and $\hat{s}$ is a suitable skewness estimator. In Table 5 we compare, for each density considered, the AE of the test (asymptotically) chosen by the Adaptive tests $A(\hat{t})$ and $B(\hat{S})$. In that table the respective larger AE-value is underlined.

For most of the classical densities considered the Adaptive test $B(\hat{S})$ based on linear rank statistics has slightly higher asymptotic power than the new test $A(\hat{t})$. The same is true for the densities of the L-DE family (not included in Table 5). For densities with larger tails and for the majority of 
the contaminated normal densities the U-statistics based test $A(\hat{t})$ is asymptotically better. However, the differences in asymptotic efficacies are small in most cases. For the contaminated normal and for the very skew densities defined on the whole real line the new adaptive test is clearly better.

\section{Simulation study}

In order to assess whether the asymptotic theory can also be applied for medium to small sample sizes a simulation study (10,000 replications each for the null case, 1,000 replications each for the alternative cases) is performed. We choose the following six distributions:

- Uniform distribution (density with small tailweight),

- Normal distribution (density with medium tailweight),

- Logistic distribution (density with medium tailweight),

- Doubleexponential distribution (density with large tailweight),

- Cauchy distribution (density with very large tailweight),

- Gumbel distribution (skew density)

We consider the four single U-tests $U_{8,1}, U_{5,1}, U_{2,1}, U_{5,2}$, and the Adaptive test $A(\hat{t})$. (Results for the Klotz-test may be obtained, e.g. from Kössler (1994), they are in accordance with the asymptotic results.) The sample sizes $n_{1}=n_{2}=10,20,40,100$ and the alternatives $\theta_{N}=N^{-1 / 2} \theta$ with various $\theta$ are considered. Estimated levels of significance are summarized in Table 6 for the uniform density. For the other densities we get very similar values. The test $U_{8: 1}$ has the largest levels. That is why we have, for short-tail densities, for the adaptive tests also relatively large values. For densities with longer tails these values are slightly smaller (about 0.02 for $n_{1}=n_{2}=10$ and 0.01 for $n_{1}=n_{2}=40$ ).

Table 6 about here

As we see from Table 6 all the tests are, for $n_{1}=n_{2} \leq 100$ slightly anticonservative. For $n_{1}=n_{2}=100$ the level is almost always less than 0.053 , and therefore $\epsilon=0.1$-robust in almost all cases. (For the notion of $\epsilon$-robustness see e.g. Rasch, Teuscher, and Guiard (2007).) 
For $n_{1}=n_{2}=10,20,40$ almost all tests are more or less anticonservative, for $n_{1}=n_{2}=10$ the estimated attained level of significance is always in $(0.085,0.125)$, for $n_{1}=n_{2}=20$ it is always in $(0.063,0.085)$, and for $n_{1}=$ $n_{2}=40$ it is always in $(0.053,0.068)$, For $n_{1}=n_{2}=100$ the results of the simulation study are summarized in Figures 3 and 4. Again, the blue dotted line is for $U_{8: 1}$, the violet short-dashed line for $U_{5: 1}$, the green long-dashed line for $U_{2: 1}$, the red dashed-dotted line for $U_{5: 2}$ and the continuous line is for the Adaptive test $A(\hat{S})$. At first we see that, for $n_{1}=n_{2}=100$, the finite power is well approximated by the asymptotic power. Moreover, it can be seen that, for a given density, there is always, sometimes together with another test, a single test which is the best. The test $U_{8: 1}$ is the best for the uniform and for the normal (together with the Adaptive test $A(\hat{t})$ ) the test $U_{5: 1}$ is the best for the logistic density (together with the Adaptive test $A(\hat{t}))$, and the test $U_{5: 2}$ is the best for the double exponential and for the Cauchy (again together with the Adaptive test $A(\hat{t}))$. All these facts are not surprising. Also, not surprisingly, that all the tests, except the adaptive test, may be bad for some densities. The Adaptive test $A(\hat{t})$ is, over all densities, the best.

Although the tests are slightly anticonservative for smaller sample sizes the order of the tests is almost the same as in the asymptotic case. For small sample sizes, $n_{1}=n_{2} \leq 20$ the adaptive test has slightly lower power as expected because of the higher misclassification probabilities.

Insert Figures 3 and 4 about here

\section{Data example}

In this section we give a data example to illustrate our adaptive procedure.

Example (cf. Example 9 of Büning, 1991). In a statistics lesson variances of median and mean are estimated for the Doubleexponential by two independent groups of students of size 24 each. Figure 7 gives a box plot of these data.

Insert Figure 5 about here 
At first, we estimate the tailweight, cf. (1), and we obtain $\hat{t}=1.4916$. According to our adaptive procedure the test $U_{8: 1}$ is chosen. We obtain $U_{8: 1}=-3.401$ an one-sided p-value of 0.0003 , a result which is, of course, not surprising. Because of the very low p-value it is no problem that the (adaptive) procedure is slightly anticonservative for the given sample size.

\section{Conclusions}

What are the results of our study? At first, we see that the finite power of the considered tests based on U-statistics can be well approximated by their asymptotic power. However, the 'convergence' of the finite power to the asymptotic power is slower than in the location case. Second, there are modifications of the "classical" Klotz test that may have (considerably) higher power than Klotz for symmetric as well asymmetric densities. Third, the Adaptive test $A(\hat{S})$ is a serious alternative to the Klotz test or to the Mood test $U_{2: 1}$ for moderate to large sample sizes, if the densities have very short tails or very long tails or if they are skew. Also, for short-tail or for long-tail densities or for skew densities the adaptive test considered here may be better than the adaptive test $B(\hat{S})$ based on linear rank tests.

Many tests based on U-Statistics have good power properties. They can be applied to a broader class of underlying densities than a linear rank test with fixed scores if the sample sizes are moderate to large.

For moderate to large sample sizes (about $n_{1}, n_{2} \geq 100$ ) the recommendation for the practising statistician is as follows: If the density is known to be exactly normal take the $F$-test. If it is only known that it has medium tails then take the Klotz test or the test $U_{5: 1}$. For densities with short tails take the test $U_{8: 1}$, and for those with long tails the test $U_{2: 1}$. If the density is known to be defined on a half interval only and is skew, then take a location test. For a completely unknown density take the adaptive test $A(\hat{S})$.

For relatively small sample sizes (about $n_{1}, n_{2} \leq 10$ ) take the Klotz test.

Note that we considered the scale problem for continuous data only. How the procedures behave for ordinal data may be a topic for further studies. Further investigations are also desirable for more general alternatives, especially for the case of unknown and unequal locations or for the case of $F \neq G$. 


\section{References}

Büning, H. (1991). Robuste und adaptive Tests. De Gruyter, Berlin.

Büning, H. (1994). Robust and adaptive tests for the two-sample location problem. OR Spektrum, 16:33-39.

Deshpande, J.V., Kochar, S.C. (1982). Some competitors of Wilcoxon-MannWhitney test for location alternatives. Journal of Indian Statistical Association, 19:9-18.

Deshpande, J.V., Kusum, K. (1984). A test for the nonparametric twosample scale problem, Australian Journal of Statistics 26 (1), 16-24.

Gibbons, J.D., Chakraborti, S. (1992). Nonparametric statistical inference, Dekker, New York.

Hájek, J., Šidák, Z., Sen, P.K. (1999). Theory of Rank Tests, San Diego: Academic Press.

Hall, D.L., Joiner, B.L. (1983). Asymptotic Relative Efficiency of R-estimators of Location. Communications in statistics, Theory and Methods, 12:739763.

Hall, P., Padmanabhan, A.R. (1997). Adaptive inference for the two-sample scale problem, Technometrics 39, No.4, 412-422.

Hogg, R.V. (1974). Adaptive robust procedures: partial review and some suggestions for future applications and theory. Journal of the American Statistical Association, 69:909-923.

Hogg, R.V. (1982). On adaptive statistical inference. Communications in Statistics, Theory and Methods, 11:2531-2542.

Hogg, R.V., Fisher, D.M., Randles, R.H. (1975). A two-sample adaptive distribution-free test. Journal of the American Statistical Association, 70:656-661.

John, M., Priebe, C.E. (2007). A data-adaptive methodology for finding an optimal weighted generalized Mann-Whitney-Wilcoxon statistic. Computational Statistics \& Data Analysis, 51:4337-4353.

Joshi, V.B. and Shetty, I.D. (2008). A note on Rosenbaum's test for twosample scale problem, Model Assisted Statistics and Applications 3, 59-69.

Kochar, S.C., Gupta, R.P. (1986). Some competitors of the Mood test for the 
two-sample scale problem. Comm.Stat., Theory Methods 15(1), 231-239.

Kössler, W. (1994). Restrictive adaptive tests for the treatment of the twosample scale problem, Computational Statistics and Data Analysis 18, 513-524.

Kössler, W. and Kumar, N. (2008). An adaptive test for the two-sample location problem based on U-statistics. Communications in Statistics Computation and Simulation, Vol. 37, 2008, 1329-1346.

Kumar, N. (1997). A Class of Two-Sample tests for Location Based on SubSample Medians. Communications in Statistics, Theory and Methods, 26:943-951.

Kumar, N. (2008). A General Class of Selection Procedures. Invited talk in the Symposium on Ranking and Selection Methodologies-Multiple Comparison Procedures during Pre-ICM International Convention on Mathematical Sciences, University of Delhi, December 18-20, 2008, submitted for publication in: EDJ-Festschrift volume in honor of E.J.Dudewicz.

Kumar, N., Singh, R.S., Öztürk, Ö. (2003). A New Class of Distribution-Free Tests for Location parameters. Sequential Analysis, 22:107-128.

Kusum, K. (1985). A new distribution-free test for the two-sample scale problem, J. Indian Statistical Assoc. 23, 97-107.

Nadarajah, S. (2009). The skew-logistic distribution, Advances in Statistical Analysis 93,2, 187-204.

Noether, G.E. (1955). On a Theorem of Pitman. Annals of Mathematical Statistics, 26:64-68.

Policello, G.E., Hettmansperger, T.P. (1976). Adaptive robust procedures for the one-sample location problem. Journal of the American Statistical Association, 71:624-633.

Ramberg, J.S., Schmeiser, B.W. (1972). An approximate method for generating symmetric random variables, Communications of the ACM, 11:987990 .

Ramberg, J.S., Schmeiser, B.W. (1974). An approximate method for generating asymmetric random variables, Communications of the ACM, 17:78-82.

Rasch, D., Teuscher, F, and Guiard, V. (2007). How robust are tests for two independent samples? Journal of Statistical Planning and Inference, 
Shetty, I.D., Govindarajulu, Z. (1988). A Two-Sample Test for Location. Communications in Statistics, Theory and Methods, 27:2389-2401.

Shetty, I.D., Pandit, P.V. (2004). A note on a new class of distributionfree tests for the two-sample scale problem based on subsample medians, Comm.Stat., theory Methods 33 (9), 2271-2280.

Sukhatme, B.V. (1957). On certain two-sample nonparametric tests for variances. AMS 28, 188-194.

Sukhatme, B.V. (1958). A two-sample distribution-free test for comparing variances. Biometrika 45, 544-548.

Tamura, R. (1960). On the nonparametric tests based on certain U-statistics. Bull.Math.Stat. 9, 61-67.

Tamura, R. (1962). On the efficiency of Sukhatme's test. Bull.Math.Stat. 10, No.3-4, 31-38.

Tamura, R. (1966). Nonparametric tests for scale. Bull.Math.Stat. 12, No.12, 89-94.

Xie, J., Priebe, C.E. (2000). Generalizing the Mann-Whitney-Wilcoxon Statistic. Nonparametric Statistics, 12:661-682.

Xie, J., Priebe, C.E. (2002). A weighted generalization of the Mann-WhitneyWilcoxon Statistic. Journal of Statistical Planning and Inference, 102:441466. 
Figure 1: The first two principal components

Figure 2: Adaptive scheme.
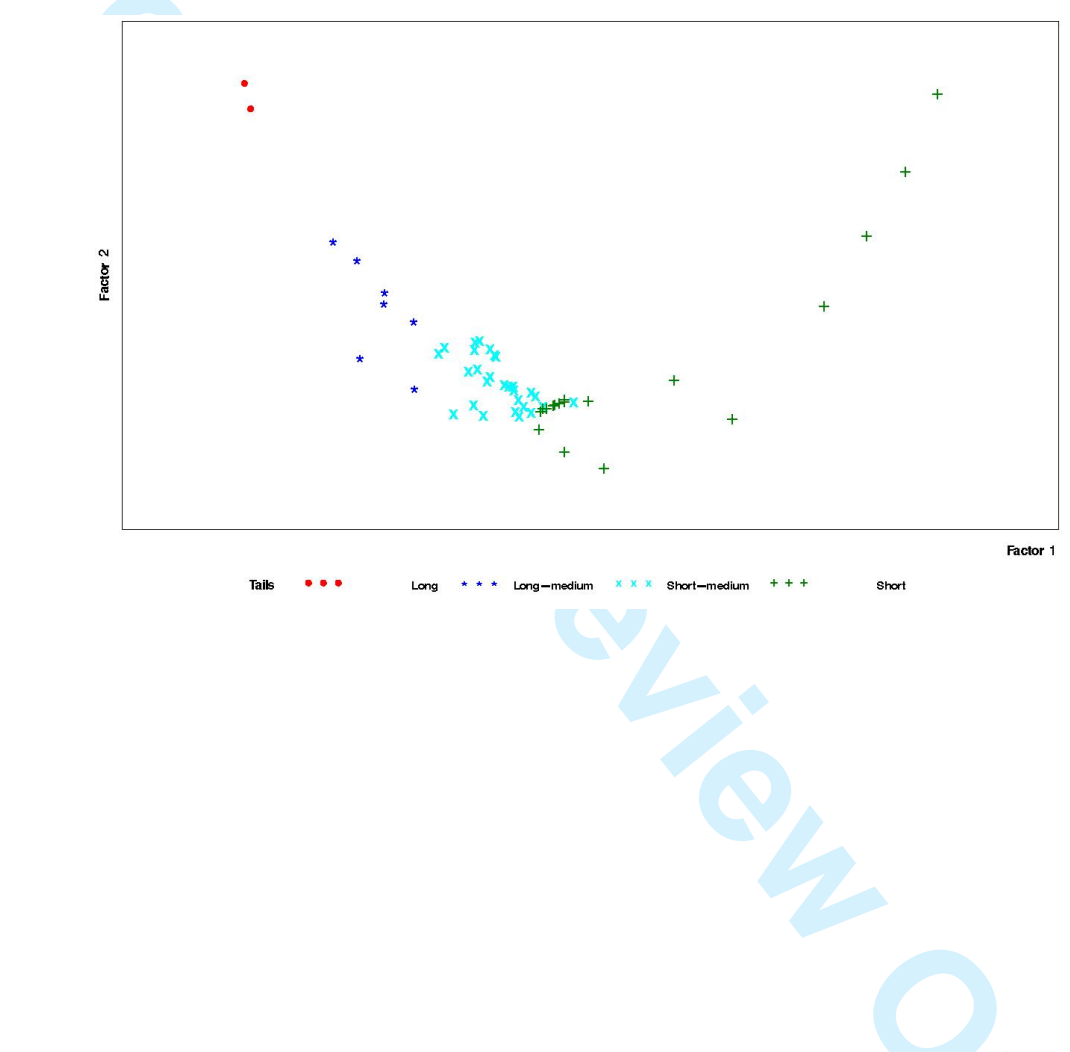

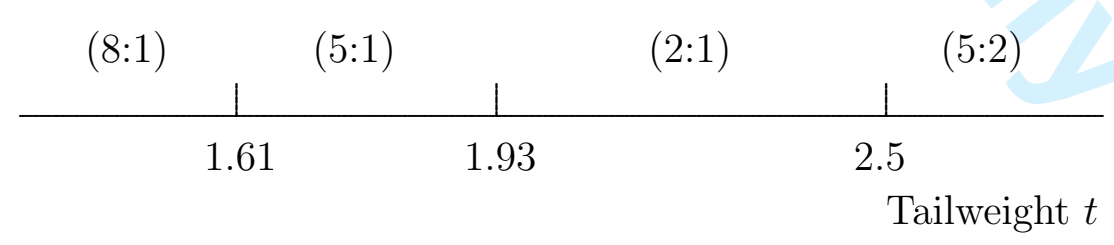


Figure 3: The asymptotic and finite $\left(n_{1}=n_{2}=100\right)$ power functions of the tests $U_{2: 1}, U_{5: 1}, U_{8: 1}, U_{5: 2}$ and $A(\hat{t})$; densities: uniform, normal and logistic.

asymptotic

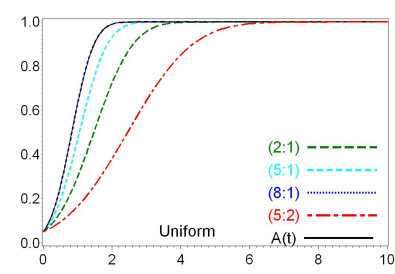

asymptotic

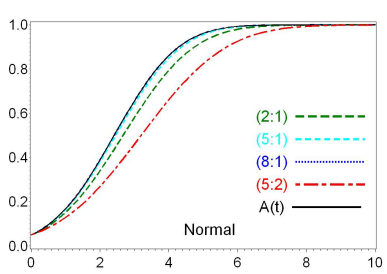

asymptotic

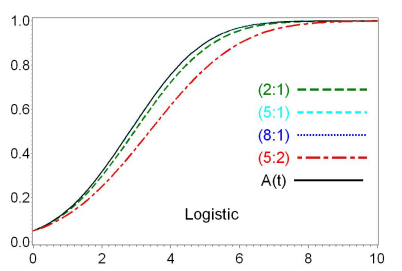

finite

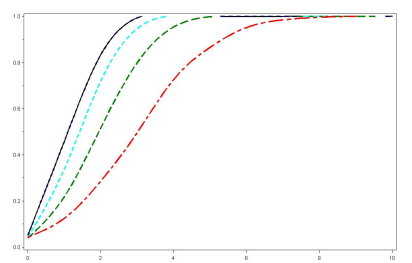

finite

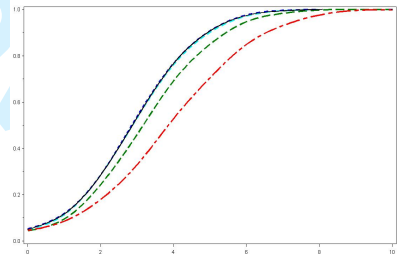

finite

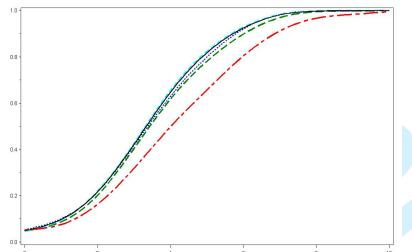


Figure 4: The asymptotic and finite $\left(n_{1}=n_{2}=100\right)$ power functions of the tests $U_{2: 1}, U_{5: 1}, U_{8: 1}, U_{5: 2}$ and $A(\hat{t})$; densities: doubleexponential, Cauchy and exponential (Continuation from Figure 3.)

asymptotic

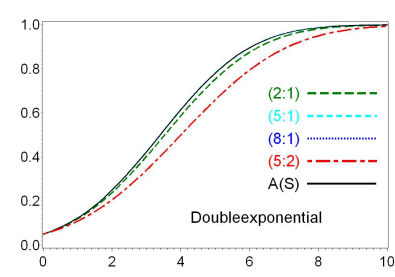

asymptotic

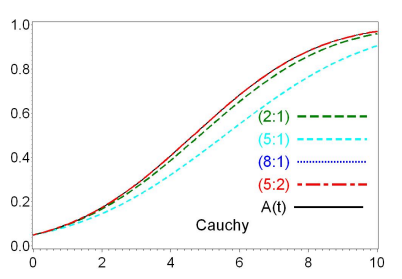

asymptotic

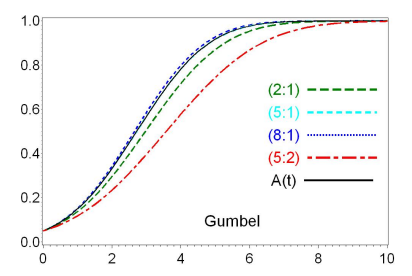

finite

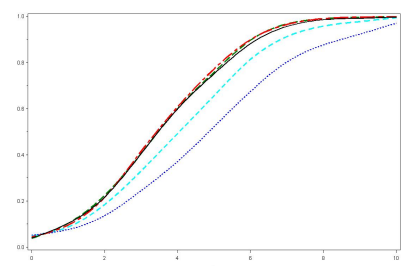

finite

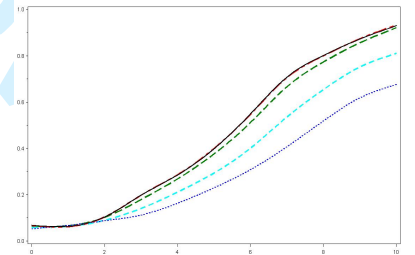

finite

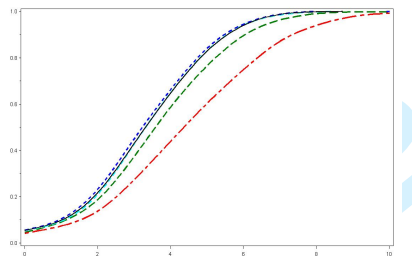


Figure 5: Boxplot of the median and mean estimates data

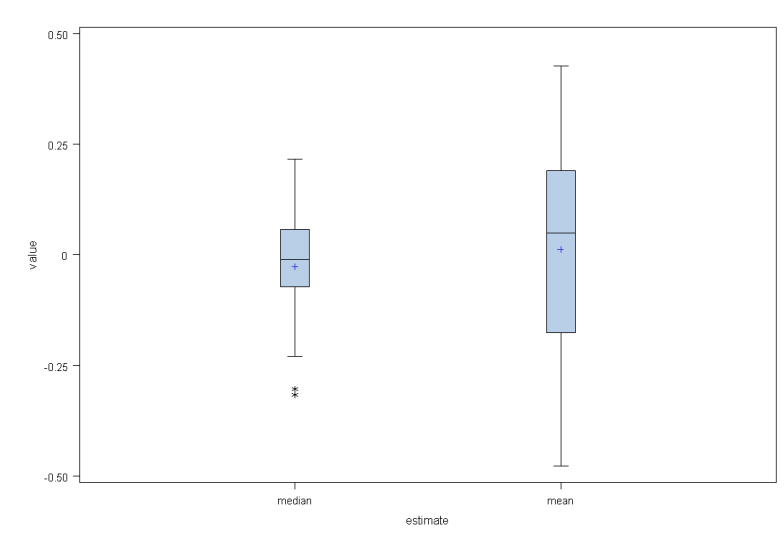


Table 1: The asymptotic efficacies for various U-tests and various densities

\begin{tabular}{|c|c|c|c|c|c|c|c|c|}
\hline density & Sukhatme & Kusum & $\begin{array}{c}\mathrm{SJ}(5,1) \\
U_{3: 1}\end{array}$ & $\mathrm{SJ}(5,2)^{*}$ & $\begin{array}{c}\mathrm{SJ}(9,1) \\
U_{5: 1}\end{array}$ & $U_{8: 1}$ & $U_{2: 1}$ & $U_{5: 2}$ \\
\hline Uniform & 12.00 & 12.44 & 6.222 & 2.333 & 9.800 & 15.66 & 5.000 & 1.802 \\
\hline Logistic & 1.040 & 1.333 & 1.307 & 1.037 & 1.372 & & 1.250 & 0.947 \\
\hline Cauchy & 0.493 & 0.407 & 0.435 & 0.496 & 0.350 & & 0.462 & 0.500 \\
\hline Doubleex & 0.75 & 0.913 & 0.902 & 0.676 & 0.936 & & 0.868 & 0.676 \\
\hline Normal & 1.216 & 1.681 & 1.624 & 1.187 & 1.795 & 1.862 & 1.520 & 1.061 \\
\hline Gumbel & 1.224 & & 1.316 & 0.950 & 1.467 & 1.537 & 1.228 & 0.847 \\
\hline
\end{tabular}


Table 2: The asymptotic efficacies of the $U_{k: i}$-tests and the corresponding factor for the Klotz-test for the scale problem

\begin{tabular}{|c|c|c|c|c|c|c|c|c|c|c|}
\hline density & Klotz & $U_{2: 1}$ & $U_{3: 1}$ & $U_{4: 1}$ & $U_{4: 2}$ & $U_{5: 1}$ & $U_{5: 2}$ & $U_{6: 1}$ & $U_{6: 2}$ & $U_{6: 3}$ \\
\hline uniform & $\infty$ & 5.000 & 6.222 & 7.936 & 1.583 & 9.800 & 1.802 & 11.735 & 2.160 & 1.020 \\
\hline logistic & 1.396 & 1.250 & 1.307 & 1.353 & 0.890 & 1.372 & 0.947 & 1.371 & 1.026 & 0.708 \\
\hline Cauchy & 0.277 & 0.462 & 0.435 & 0.394 & 0.497 & 0.350 & 0.500 & 0.311 & 0.496 & 0.468 \\
\hline $\mathrm{DE}$ & 0.997 & 0.868 & 0.902 & 0.927 & 0.641 & 0.936 & 0.676 & 0.933 & 0.722 & 0.526 \\
\hline normal & 2.000 & 1.520 & 1.624 & 1.727 & 0.987 & 1.795 & 1.061 & 1.837 & 1.169 & 0.759 \\
\hline Gumbel & 1.670 & 1.228 & 1.316 & 1.406 & 0.786 & 1.467 & 0.847 & 1.506 & 0.935 & 0.603 \\
\hline skew-log_1 & 0.804 & 1.089 & 1.149 & 1.204 & 0.745 & $\underline{1.233}$ & 0.797 & 1.245 & 0.870 & 0.584 \\
\hline skew-log_2 & & 0.788 & 0.841 & 0.892 & 0.216 & $\underline{0.925}$ & 0.206 & 0.943 & & \\
\hline$t_{1.5}$ & 0.454 & 0.659 & 0.641 & 0.605 & 0.626 & 0.560 & 0.642 & 0.515 & 0.658 & 0.555 \\
\hline$t_{2}$ & 0.610 & 0.800 & 0.794 & 0.768 & 0.700 & 0.729 & 0.730 & 0.686 & 0.761 & 0.604 \\
\hline$t_{3}$ & 0.853 & 0.983 & 0.997 & 0.993 & 0.790 & $\underline{0.968}$ & 0.829 & 0.934 & 0.880 & 0.654 \\
\hline$t_{4}$ & 1.030 & 1.093 & 1.122 & 1.135 & 0.837 & $\underline{1.124}$ & 0.884 & 1.100 & 0.947 & 0.681 \\
\hline$t_{5}$ & 1.163 & 1.166 & 1.207 & 1.232 & 0.866 & $\underline{1.232}$ & 0.918 & 1.215 & 0.989 & 0.696 \\
\hline$t_{10}$ & 1.509 & 1.330 & 1.398 & 1.457 & 0.925 & $\underline{1.485}$ & 0.988 & 1.491 & & \\
\hline $\mathrm{RST}_{-}-1.0$ & 0.249 & 0.414 & 0.389 & 0.351 & 0.450 & 0.313 & 0.451 & 0.278 & 0.445 & 0.429 \\
\hline $\mathrm{RST}_{-}-0.5$ & 0.508 & 0.684 & 0.672 & 0.643 & 0.628 & 0.604 & 0.646 & 0.563 & 0.665 & 0.553 \\
\hline $\mathrm{RST}_{-}-0.4$ & 0.603 & 0.765 & 0.760 & 0.737 & 0.673 & 0.701 & 0.697 & 0.662 & 0.724 & 0.582 \\
\hline $\mathrm{RST}_{-}-0.3$ & 0.726 & 0.859 & 0.863 & 0.850 & 0.722 & 0.820 & 0.752 & 0.785 & 0.789 & 0.612 \\
\hline $\mathrm{RST}_{-}-0.2$ & 0.886 & 0.969 & 0.986 & 0.986 & 0.774 & $\underline{0.966}$ & 0.812 & 0.937 & 0.861 & 0.643 \\
\hline $\mathrm{RST}_{-}-0.1$ & 1.102 & 1.098 & 1.132 & 1.151 & 0.830 & $\underline{1.147}$ & 0.877 & 1.129 & 0.940 & 0.675 \\
\hline RST_0.05 & 1.588 & 1.336 & 1.407 & 1.471 & 0.922 & $\underline{1.504}$ & 0.984 & 1.516 & 1.073 & 0.725 \\
\hline RST_0.14 & 2.035 & 1.509 & 1.613 & 1.716 & 0.981 & $\overline{1.784}$ & 1.054 & 1.826 & 1.161 & 0.756 \\
\hline RST_0.2 & 2.435 & 1.641 & 1.771 & 1.906 & 1.022 & 2.006 & 1.103 & 2.075 & 1.223 & 0.777 \\
\hline RST_0.4 & 4.948 & 2.188 & 2.446 & 2.750 & 1.166 & 3.015 & 1.278 & 3.238 & 1.449 & 0.846 \\
\hline L-DE_0.55 & 0.977 & 0.872 & 0.905 & 0.931 & 0.645 & 0.938 & 0.680 & 0.936 & 0.726 & 0.530 \\
\hline L-DE_0.61 & 0.986 & 0.888 & 0.920 & 0.944 & 0.663 & 0.950 & 0.697 & 0.946 & 0.743 & 0.548 \\
\hline L-DE_0.7 & 1.025 & 0.939 & 0.969 & 0.989 & 0.714 & 0.991 & 0.749 & 0.984 & 0.795 & 0.595 \\
\hline L-DE_0.75 & 1.061 & 0.983 & 1.013 & 1.031 & 0.752 & 1.031 & 0.789 & 1.020 & 0.837 & 0.626 \\
\hline L-DE_0.8 & 1.106 & 1.037 & 1.069 & 1.087 & 0.793 & 1.085 & 0.833 & 1.071 & 0.887 & 0.655 \\
\hline L-DE_0.9 & 1.241 & 1.166 & 1.208 & 1.236 & 0.863 & 1.239 & 0.914 & 1.226 & 0.984 & 0.695 \\
\hline L-DE_0.95 & 1.328 & 1.223 & 1.274 & 1.313 & 0.884 & 1.324 & 0.939 & 1.318 & 1.015 & 0.705 \\
\hline L-DE_0.97 & 1.361 & 1.239 & 1.294 & 1.337 & 0.888 & 1.352 & 0.944 & 1.348 & 1.022 & 0.707 \\
\hline L-DE_0.99 & 1.388 & 1.249 & 1.305 & 1.351 & 0.890 & 1.369 & 0.947 & 1.368 & 1.026 & 0.708 \\
\hline
\end{tabular}


continuation from previuos page

\begin{tabular}{|c|c|c|c|c|c|c|c|c|c|c|}
\hline density & Klotz & $U_{2: 1}$ & $U_{3: 1}$ & $U_{4: 1}$ & $U_{4: 2}$ & $U_{5: 1}$ & $U_{5: 2}$ & $U_{6: 1}$ & $U_{6: 2}$ & $U_{6: 3}$ \\
\hline U-L_0.55 & 1.481 & 1.331 & 1.393 & 1.443 & 0.945 & 1.463 & 1.007 & 1.462 & 1.093 & 0.749 \\
\hline U-L_0.61 & 1.602 & 1.471 & 1.542 & 1.599 & 1.034 & 1.623 & 1.105 & 1.622 & 1.204 & 0.811 \\
\hline U-L_0.7 & 2.016 & 1.798 & 1.900 & 1.989 & 1.207 & 2.032 & 1.303 & 2.040 & 1.443 & 0.912 \\
\hline U-L_0.75 & 2.377 & 2.061 & 2.200 & 2.331 & 1.313 & 2.404 & 1.432 & 2.431 & 1.608 & 0.959 \\
\hline U-L_0.8 & 2.925 & 2.402 & 2.605 & 2.813 & 1.412 & 2.947 & 1.559 & 3.019 & 1.783 & 0.992 \\
\hline U-L_0.9 & 5.595 & 3.398 & 3.886 & 4.472 & 1.552 & 4.975 & 1.753 & 5.375 & 2.077 & 1.018 \\
\hline U-L_0.95 & 9.585 & 4.106 & 4.879 & 5.882 & 1.578 & 6.854 & 1.794 & 7.743 & 2.147 & 1.020 \\
\hline U-L_0.97 & 13.62 & 4.439 & 5.368 & 6.612 & 1.582 & 7.874 & 1.800 & 9.090 & 2.157 & 1.020 \\
\hline U-L_0.99 & 25.10 & 4.794 & 5.888 & 7.387 & 1.586 & 8.958 & 1.807 & 10.519 & 2.169 & 1.020 \\
\hline CN_2,0.01 & 1.861 & 1.503 & 1.602 & 1.699 & 0.983 & 1.762 & 1.057 & 1.797 & 1.163 & 0.758 \\
\hline CN_2,0.02 & 1.782 & 1.486 & 1.581 & 1.673 & 0.980 & 1.730 & 1.053 & 1.760 & 1.158 & 0.756 \\
\hline CN_2,0.05 & 1.610 & 1.439 & 1.523 & 1.599 & 0.969 & $\underline{1.641}$ & 1.040 & 1.658 & 1.141 & 0.751 \\
\hline CN_2,0.10 & 1.449 & 1.370 & 1.438 & 1.495 & 0.952 & $\overline{1.519}$ & 1.018 & 1.519 & 1.113 & 0.743 \\
\hline CN_3,0.01 & 1.711 & 1.485 & 1.579 & 1.670 & 0.981 & 1.725 & 1.053 & 1.753 & 1.159 & 0.757 \\
\hline CN_3,0.02 & 1.569 & 1.451 & 1.537 & 1.615 & 0.974 & 1.659 & 1.045 & 1.676 & 1.148 & 0.754 \\
\hline CN_3,0.05 & 1.292 & 1.358 & 1.420 & 1.469 & 0.955 & 1.483 & 1.021 & 1.473 & 1.116 & 0.745 \\
\hline CN_3,0.10 & 1.094 & 1.225 & 1.259 & 1.274 & 0.921 & $\underline{1.257}$ & 0.980 & 1.221 & 1.062 & 0.730 \\
\hline CN_5,0.01 & 1.560 & 1.466 & 1.554 & 1.637 & 0.978 & $\overline{1.684}$ & 1.050 & 1.704 & 1.154 & 0.755 \\
\hline CN_5,0.02 & 1.403 & 1.414 & 1.489 & 1.553 & 0.969 & $\underline{1.582}$ & 1.038 & 1.584 & 1.139 & 0.752 \\
\hline CN_5,0.05 & 1.075 & 1.273 & 1.313 & 1.334 & 0.940 & 1.321 & 1.003 & 1.286 & 1.091 & 0.739 \\
\hline CN_5,0.10 & 0.841 & 1.079 & 1.082 & 1.058 & 0.888 & 1.009 & 0.938 & 0.946 & 1.006 & 0.717 \\
\hline CN_10,0.01 & 1.387 & 1.449 & 1.533 & 1.609 & 0.976 & 1.649 & 1.047 & 1.662 & 1.150 & 0.755 \\
\hline CN_10,0.02 & 1.318 & 1.382 & 1.447 & 1.500 & 0.964 & 1.516 & 1.033 & 1.507 & 1.131 & 0.750 \\
\hline CN_10,0.05 & 0.904 & 1.201 & 1.224 & 1.223 & 0.927 & $\underline{1.189}$ & 0.986 & 1.136 & 1.069 & 0.734 \\
\hline CN_10,0.10 & 0.685 & 0.961 & 0.942 & 0.894 & 0.858 & 0.825 & 0.901 & 0.749 & 0.956 & 0.705 \\
\hline RST-.4,-..49 & 0.585 & 0.718 & 0.710 & 0.686 & 0.640 & 0.650 & 0.661 & 0.612 & 0.685 & 0.556 \\
\hline RST-.2,-.49 & 0.595 & $\underline{0.646}$ & 0.661 & 0.665 & 0.502 & 0.655 & 0.530 & 0.638 & 0.566 & 0.409 \\
\hline RST-.2,-.4 & 0.678 & $\underline{0.751}$ & 0.764 & 0.765 & 0.596 & 0.750 & 0.626 & 0.726 & 0.666 & 0.492 \\
\hline $\mathrm{RST}_{-} .2, .4$ & 3.415 & 1.898 & 2.094 & 2.316 & 1.072 & 2.500 & 1.169 & 2.648 & 1.316 & 0.790 \\
\hline $\mathrm{RST}_{.} .2, .49$ & 4.222 & 2.084 & 2.327 & 2.611 & 1.117 & 2.868 & 1.224 & 3.065 & 1.387 & 0.811 \\
\hline $\mathrm{RST}_{-} .4, .49$ & 3.962 & 2.353 & 2.654 & 3.016 & 1.203 & 3.342 & 1.324 & 3.624 & 1.509 & 0.863 \\
\hline
\end{tabular}


Table 3: The asymptotic efficacies of the tests $U_{8: 1}$ and $U_{5: 1}$ for the scale problem, short tail densities only

\begin{tabular}{lccc|lccc|} 
density & tailweight & $U_{8: 1}$ & $U_{5: 1}$ & density & tailweight & $U_{8: 1}$ & $U_{5: 1}$ \\
\hline uniform & 1.286 & $\mathbf{1 5 . 6 6}$ & 9.800 & U-L_0.55 & 1.668 & 1.425 & 1.463 \\
normal & 1.587 & $\mathbf{1 . 8 6 2}$ & 1.795 & U-L_0.61 & 1.623 & 1.580 & 1.623 \\
Gumbel & 1.655 & $\mathbf{1 . 5 3 7}$ & 1.467 & U-L_0.7 & 1.534 & $\underline{1.993}$ & 2.032 \\
RST_0.05 & 1.657 & 1.499 & 1.504 & U-L_0.75 & 1.474 & $\underline{2.393}$ & 2.404 \\
RST_0.14 & 1.591 & $\mathbf{1 . 8 5 4}$ & 1.784 & U-L_0.8 & 1.409 & $\mathbf{3 . 0 2 7}$ & 2.947 \\
RST_0.2 & 1.552 & $\mathbf{2 . 1 4 6}$ & 2.006 & U-L_0.9 & 1.300 & $\mathbf{5 . 8 8 8}$ & 4.945 \\
RST_0.4 & 1.446 & $\mathbf{3 . 5 7 6}$ & 3.015 & U-L_0.95 & & $\mathbf{9 . 2 1 4}$ & 6.854 \\
& & & & U-L_0.97 & & $\mathbf{1 1 . 2 8}$ & 7.874 \\
& & & & U-L_0.99 & & $\mathbf{1 1 . 6 8}$ & 8.958 \\
CN_2,0.01 & 1.592 & $\mathbf{1 . 8 1 2}$ & 1.762 & CN_2,0.02 & 1.597 & $\mathbf{1 . 7 6 5}$ & 1.730 \\
CN_2,0.05 & 1.611 & 1.639 & 1.641 & CN_2,0.10 & 1.636 & 1.470 & 1.519 \\
CN_3,0.01 & 1.596 & $\mathbf{1 . 7 5 5}$ & 1.725 & CN_3,0.02 & 1.605 & $\underline{1.655}$ & 1.659 \\
CN_3,0.05 & 1.635 & 1.400 & 1.483 & CN_3,0.10 & 1.697 & 1.086 & 1.257 \\
CN_5,0.01 & 1.600 & $\mathbf{1 . 6 9 0}$ & 1.684 & CN_5,0.02 & 1.614 & 1.535 & 1.582 \\
CN_5,0.05 & 1.665 & 1.154 & 1.321 & CN_5,0.10 & 1.974 & 0.728 & 1.009 \\
CN_10,0.01 & 1.603 & $\underline{1.633}$ & 1.649 & CN_10,0.02 & 1.622 & 1.433 & 1.516 \\
CN_10,0.05 & 1.694 & 0.966 & 1.189 & CN_10,0.10 & 1.944 & 0.498 & 0.825 \\
skew-log_1 & 1.672 & 1.233 & 1.233 & & & & \\
skew-log_2 & 1.684 & & 0.925 & & & & \\
RST_0.2,0.4 & 1.546 & $\mathbf{2 . 8 5 2}$ & 2.500 & & & & \\
RST_0.2,0.49 & 1.507 & $\mathbf{3 . 3 7 4}$ & 2.868 & & & & \\
RST_0.4,0.49 & 1.456 & $\mathbf{4 . 0 7 0}$ & 3.342 & & & &
\end{tabular}

Table 4: The AE of the tests $U_{k: i}$, for the scale problem, together with that for the Mann-Whitney test $U_{1,1}$, skew densities with $F(0)=0$.

\begin{tabular}{lllllllllll} 
density & $U_{1,1}$ & $U_{2: 1}$ & $U_{3: 1}$ & $U_{4: 1}$ & $U_{4: 2}$ & $U_{5: 1}$ & $U_{5: 2}$ & $U_{6: 1}$ & $U_{6: 2}$ & $U_{6: 3}$ \\
\hline exponential & 0.750 & 0.139 & 0.148 & 0.157 & 0.092 & 0.162 & 0.098 & 0.165 & 0.108 & 0.071 \\
Gamma1.5 & 1.216 & 0.150 & 0.159 & 0.169 & 0.098 & 0.175 & 0.105 & 0.179 & 0.116 & 0.076 \\
Gamma2 & 1.688 & 0.155 & 0.165 & 0.175 & 0.101 & 0.182 & 0.109 & 0.185 & 0.120 & 0.078 \\
Gamma2.5 & 2.162 & 0.158 & 0.168 & 0.179 & 0.103 & 0.185 & 0.111 & 0.189 & 0.122 & 0.080 \\
Gamma3 & 2.637 & 0.160 & 0.170 & 0.181 & 0.104 & 0.188 & 0.112 & 0.192 & 0.123 & 0.080 \\
Gamma4 & 3.589 & 0.162 & 0.173 & 0.184 & 0.106 & 0.191 & 0.114 & 0.195 & 0.125 & 0.081 \\
Gamma5 & 4.542 & 0.164 & 0.175 & 0.186 & 0.107 & 0.193 & 0.115 & 0.197 & 0.126 & 0.082 \\
LogNormal & 0.955 & 0.0 & 0.0 & 0.0 & 0.0 & 0.0 & 0.0 & 0.0 & 0.0 & 0.0
\end{tabular}


Table 5: The test chosen by the Adaptive tests $A(\hat{t})$ and $B(\hat{S})$ and their factors $C_{k, i}^{2}(f)$

\begin{tabular}{|c|c|c|c|c|c|}
\hline \multirow[b]{2}{*}{ density } & \multirow[b]{2}{*}{ tailweight } & \multicolumn{2}{|c|}{ Adaptive test $A(\hat{t})$} & \multicolumn{2}{|c|}{ Adaptive test $B(\hat{S})$} \\
\hline & & chosen test & $\mathrm{AE}$ & chosen test & $\mathrm{AE}$ \\
\hline uniform & 1.286 & $U_{8: 1}$ & 15.66 & Klotz & $\underline{\infty}$ \\
\hline logistic & 1.697 & $U_{5: 1}$ & 1.372 & logistic scores & 1.428 \\
\hline Cauchy & 3.217 & $U_{5: 2}$ & $\underline{0.500}$ & Ansari-Bradley & $\overline{0.498}$ \\
\hline $\mathrm{DE}$ & 1.912 & $U_{5: 1}$ & $\overline{0.936}$ & logistic scores & $\underline{0.996}$ \\
\hline Normal & 1.587 & $U_{8: 1}$ & 1.862 & Klotz & 2.000 \\
\hline$t_{1.5}$ & 2.433 & $U_{2: 1}$ & $\underline{0.659}$ & Ansari-Bradley & 0.657 \\
\hline$t_{2}$ & 2.107 & $U_{2: 1}$ & $\underline{0.800}$ & Ansari-Bradley & 0.750 \\
\hline$t_{3}$ & 1.798 & $U_{5: 1}$ & $\underline{0.968}$ & logistic scores & 0.942 \\
\hline$t_{4}$ & 1.786 & $U_{5: 1}$ & 1.124 & logistic scores & 1.111 \\
\hline$t_{5}$ & 1.737 & $U_{5: 1}$ & $\underline{1.232}$ & logistic scores & $\underline{1.232}$ \\
\hline$t_{10}$ & 1.672 & $U_{5: 1}$ & 1.485 & logistic scores & $\underline{1.537}$ \\
\hline $\mathrm{RST}_{-}-1$ & 3.451 & $U_{5: 2}$ & $\underline{0.451}$ & Ansari-Bradley & $\overline{0.448}$ \\
\hline $\mathrm{RST}_{-}-0.5$ & 2.302 & $U_{2: 1}$ & $\underline{0.684}$ & Ansari-Bradley & 0.661 \\
\hline $\mathrm{RST}_{-}-0.4$ & 2.146 & $U_{2: 1}$ & $\underline{0.765}$ & Ansari-Bradley & 0.720 \\
\hline $\mathrm{RST}_{-}-0.3$ & 2.010 & $U_{2: 1}$ & $\overline{0.859}$ & logistic scores & 0.808 \\
\hline $\mathrm{RST}_{-}-0.2$ & 1.891 & $U_{5: 1}$ & $\underline{0.966}$ & logistic scores & 0.963 \\
\hline RST_-0.1 & 1.788 & $U_{5: 1}$ & 1.147 & logistic scores & $\underline{1.164}$ \\
\hline RST_0.05 & 1.657 & $U_{5: 1}$ & 1.516 & logistic scores & $\underline{1.592}$ \\
\hline RST_0.14 & 1.591 & $U_{8: 1}$ & 1.854 & Klotz & 2.015 \\
\hline RST_0.2 & 1.552 & $U_{8: 1}$ & 2.146 & Klotz & $\underline{2.394}$ \\
\hline RST_0.4 & 1.446 & $U_{8: 1}$ & 3.576 & Klotz & 4.531 \\
\hline U-L_0.55 & 1.668 & $U_{5: 1}$ & 1.463 & logistic scores & 1.517 \\
\hline U-L_0.61 & 1.623 & $U_{5: 1}$ & $\underline{1.623}$ & Klotz & 1.602 \\
\hline U-L_0.7 & 1.534 & $U_{8: 1}$ & 1.993 & Klotz & 2.016 \\
\hline U-L_0.75 & 1.474 & $U_{8: 1}$ & $\underline{2.393}$ & Klotz & 2.377 \\
\hline U-L_0.8 & 1.409 & $U_{8: 1}$ & 3.027 & Klotz & 2.925 \\
\hline U-L_0.9 & 1.300 & $U_{8: 1}$ & 5.888 & Klotz & 5.595 \\
\hline U-L_0.95 & & $U_{8: 1}$ & 9.214 & Klotz & $\underline{9.585}$ \\
\hline U-L_0.99 & & $U_{8: 1}$ & 11.28 & Klotz & $\underline{13.62}$ \\
\hline
\end{tabular}




\begin{tabular}{|c|c|c|c|c|c|c|}
\hline \multirow[b]{2}{*}{ density } & \multirow[b]{2}{*}{ tailw. } & \multirow[b]{2}{*}{ skewn. } & \multicolumn{2}{|c|}{ Adaptive test $A(\hat{t})$} & \multicolumn{2}{|c|}{ Adaptive test $B(\hat{S})$} \\
\hline & & & chosen test & $\mathrm{AE}$ & chosen test & $\mathrm{AE}$ \\
\hline CN_2,0.01 & 1.592 & & $U_{8: 1}$ & 1.812 & Klotz & 1.861 \\
\hline CN_2,0.02 & 1.597 & & $U_{8: 1}$ & 1.765 & Klotz & $\overline{1.782}$ \\
\hline CN_2,0.05 & 1.611 & & $U_{5: 1}$ & 1.641 & Klotz & $\overline{1.610}$ \\
\hline CN_2,0.1 & 1.636 & & $U_{5: 1}$ & $\underline{1.519}$ & Klotz & 1.449 \\
\hline CN_3,0.01 & 1.596 & & $U_{8: 1}$ & $\underline{1.755}$ & Klotz & 1.711 \\
\hline CN_3,0.02 & 1.605 & & $U_{5: 1}$ & 1.655 & Klotz & 1.569 \\
\hline CN_3,0.05 & 1.635 & & $U_{5: 1}$ & $\underline{1.483}$ & Klotz & 1.292 \\
\hline CN_3,0.1 & 1.697 & & $U_{5: 1}$ & $\overline{1.257}$ & logistic scores & 1.198 \\
\hline CN_5,0.01 & 1.600 & & $U_{8: 1}$ & $\underline{1.690}$ & Klotz & 1.560 \\
\hline CN_5,0.02 & 1.614 & & $U_{5: 1}$ & $\underline{1.582}$ & Klotz & 1.403 \\
\hline CN_5,0.05 & 1.665 & & $U_{5: 1}$ & $\overline{1.321}$ & logistic scores & 1.202 \\
\hline CN_5,0.1 & 1.794 & & $U_{5: 1}$ & $\underline{1.009}$ & logistic scores & 0.956 \\
\hline CN_10,0.01 & 1.603 & & $U_{8: 1}$ & $\overline{1.634}$ & Klotz & 1.387 \\
\hline CN_10,0.02 & 1.622 & & $U_{5: 1}$ & 1.516 & Klotz & 1.318 \\
\hline CN_10,0.05 & 1.694 & & $U_{5: 1}$ & 1.189 & logistic scores & 1.044 \\
\hline CN_10,0.1 & 1.944 & & $U_{2: 1}$ & $\overline{0.961}$ & logistic scores & 0.798 \\
\hline Gumbel & 1.655 & & $U_{5: 1}$ & $\overline{1.467}$ & logistic scores & . \\
\hline skew-log1 & 1.672 & 0.1313 & $U_{5: 1}$ & 1.233 & logistic scores & 0.955 \\
\hline skew-log2 & 1.684 & 0.2279 & $U_{5: 1}$ & 0.925 & logistic scores & \\
\hline RST-0.4,-0.49 & 2.226 & 0.375 & $U_{2: 1}$ & $\underline{0.718}$ & Wilcoxon & 0.047 \\
\hline RST-0.2,-0.49 & 2.195 & 0.490 & $U_{2: 1}$ & $\overline{0.646}$ & Wilcoxon & 0.002 \\
\hline RST-0.2,-0.4 & 2.073 & & $U_{2: 1}$ & $\overline{0.751}$ & Ansari-Bradley & 0.665 \\
\hline RST_0.2,0.4 & 1.546 & & $U_{8: 1}$ & 2.852 & Klotz & $\underline{3.415}$ \\
\hline RST_0.2,0.49 & 1.507 & & $U_{8: 1}$ & 3.374 & Klotz & 4.222 \\
\hline RST_0.4,0.49 & 1.456 & & $U_{8: 1}$ & $\underline{4.070}$ & Klotz & 3.962 \\
\hline
\end{tabular}

Table 6: Estimated levels of significance for various sample sizes and for various $\mathrm{U}$-tests and for the adaptive test.

\begin{tabular}{l|ccccc} 
& $U_{2: 1}$ & $U_{5: 1}$ & $U_{8: 1}$ & $U_{5: 2}$ & Adaptive \\
\hline$n_{1}=n_{2}=10$ & 0.085 & 0.100 & 0.125 & 0.118 & 0.123 \\
$n_{1}=n_{2}=20$ & 0.065 & 0.071 & 0.085 & 0.072 & 0.085 \\
$n_{1}=n_{2}=40$ & 0.058 & 0.060 & 0.067 & 0.060 & 0.067 \\
$n_{1}=n_{2}=100$ & 0.052 & 0.051 & 0.053 & 0.053 & 0.053
\end{tabular}

